\title{
The Metal as Source of Chirality in Octahedral Complexes with Tripodal Tetradentate Ligands
}

María Carmona, Ricardo Rodríguez, Vincenzo Passarelli, Fernando J. Lahoz, Pilar García-Orduña, and Daniel Carmona

J. Am. Chem. Soc., Just Accepted Manuscript • DOI: 10.1021/jacs.7b12731 • Publication Date (Web): 04 Jan 2018

Downloaded from http://pubs.acs.org on January 5, 2018

\section{Just Accepted}

"Just Accepted" manuscripts have been peer-reviewed and accepted for publication. They are posted online prior to technical editing, formatting for publication and author proofing. The American Chemical Society provides "Just Accepted" as a free service to the research community to expedite the dissemination of scientific material as soon as possible after acceptance. "Just Accepted" manuscripts appear in full in PDF format accompanied by an HTML abstract. "Just Accepted" manuscripts have been fully peer reviewed, but should not be considered the official version of record. They are accessible to all readers and citable by the Digital Object Identifier (DOI®). "Just Accepted" is an optional service offered to authors. Therefore, the "Just Accepted" Web site may not include all articles that will be published in the journal. After a manuscript is technically edited and formatted, it will be removed from the "Just Accepted" Web site and published as an ASAP article. Note that technical editing may introduce minor changes to the manuscript text and/or graphics which could affect content, and all legal disclaimers and ethical guidelines that apply to the journal pertain. ACS cannot be held responsible for errors or consequences arising from the use of information contained in these "Just Accepted" manuscripts. 
Chiral metallic complexes constitute an important class of asymmetric catalysts that consist of assemblies of ligands around a central metal atom with the stereogenic element(s) located in the ligands and/or in the metal. The resulting chiral entity activates reagents usually by coordination to the metal atom and, in the course of the reaction, the chirality is transferred to the newly formed stereogenic centers. ${ }^{1}$ In this regard, it has been argued ${ }^{2}$ that the shorter the distance between the chiral element and the activated substrate, the more efficient the chirality transfer. Hence, chiral-at-metal catalysts look like the best candidates to accomplish efficient asymmetric induction because they offer the opportunity of achieving a direct contact between the chiral center and the substrate.

However, while asymmetric catalysis mediated by metallic compounds bearing chiral ligands is abundantly represented in the scientific literature, ${ }^{1}$ enantioselective catalytic systems based on stereogenic metals are much less documented. ${ }^{3}$ The demanding requirements of optical resolution, high configurational stability and labile coordination sites can be envisaged as responsible for this scarcity.

So far most of the reported catalysts containing stereogenic metal centers adopt half-sandwich pseudooctahedral ${ }^{4}$ or octahedral geometries., ${ }^{2,5}$ Further, the optical resolution concern is sometimes circumvented by using enantiopure chiral ligands and taking advantage of the chirality predetermination, ${ }^{6}$ whereas the use of multidentate ligands is one of the most successful approaches to address the issue of configurational stability concurrent with coordination sites lability.

We have recently developed a synthetic route for the preparation of a new type of octahedral rhodium(III) and iridium(III) complexes containing tripodal tetradentate ligands (Chart 1). We have shown that the tetracoordinate frame of the ligands maintains

the configuration at the metal ${ }^{7}$ whereas the two remaining coordination sites could be available for catalytic transformations. Notably, only the diastereomer

Chart 1. Octahedral complexes with tripodal tetradentate ligands
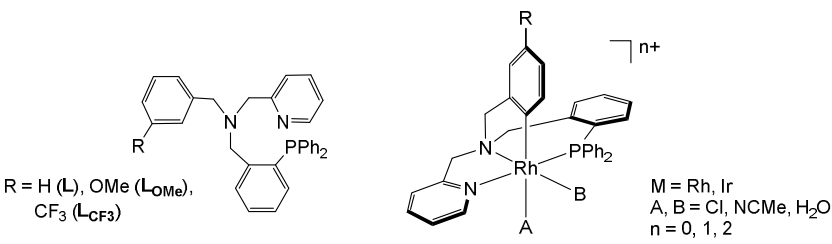

in which the phosphorus and the pyridinic nitrogen atoms are mutually trans was observed and isolated in high yield. Moreover, starting from racemic $\mathbf{L}$, only one chiral racemic topology selectively formed for all the metals and the ligands used: the sign of the chirality adopted by the metal predetermines the configuration at the aminic nitrogen. Here we report the optical resolution of the obtained rhodium racemates and the results of catalytic tests on the Diels-Alder reaction proving that the chirality is efficiently transferred from the metal to the substrate. On the basis of experimental NMR studies and DFT calculations, the activity of the catalytic systems and the origin of the enantioselectivity are discussed.

Racemic $1^{7 \mathrm{~b}}$ reacted with enantiopure $(S)$-amino carboxylates (2) in refluxing ethanol to provide $1 / 1$ diastereomeric mixtures of $(A, S)-3$ and $(C, S)-3^{8}$ (Scheme 1$)$. No kinetic resolution was observed at this step even when the reaction was carried out at lower temperatures and/or with lower amino carboxylate concentrations or when $\alpha$-amino acids such as valine, alanine, phenylglycine, tert-leucine, proline or $N$-methylproline were used.

Complexes 3 were characterized by spectroscopic and analytical methods and by the determination of the crystal structure of the phenylglycinato derivative (SI). Only the coordination isomer in which the oxygen atom of the carboxylato group is trans to the aminic nitrogen of the tetradentate ligand was isolated. As a confirmation, the diastereomers $(C, S)$-3 and $(A, S)$-3 were calculated to be more stable than the related isomers containing the oxygen atom trans to the metalated phenyl group (Figure S.6, SI). Also, confirming the lack of kinetic resolution, the four putative intermediates of formula $\left[\operatorname{Rh}\left(\kappa^{4} C, N, N^{\prime}, P-\mathbf{L}\right)\{(S)\right.$ $\left.\left.\mathrm{NH}_{2} \mathrm{CHPhCOO}\right\}(\mathrm{NCMe})\right]^{+}$exhibit similar relative stabilities thus indicating that all the reaction paths should be accessible and the 
selective formation of $(C, S)-\mathbf{3}$ and $(A, S)$-3 mainly relies on thermodynamics (Figure S.6, SI).

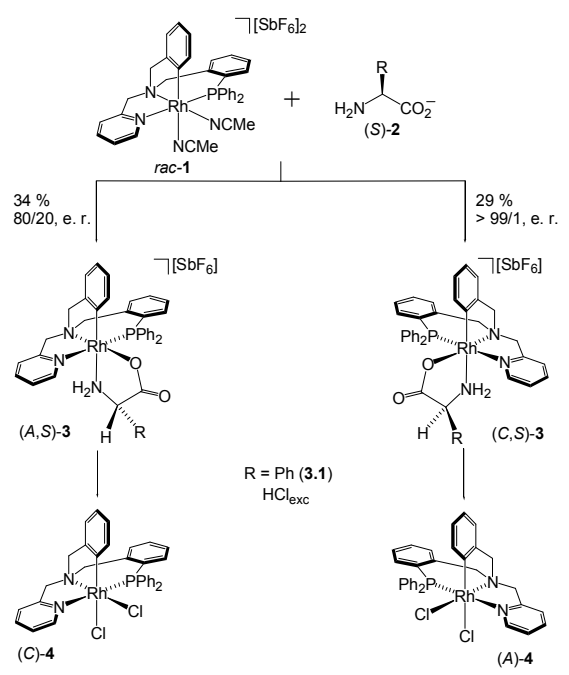

Scheme 1. (S)-Phenylglycinato mediated resolution of the dichloridos 4

The diastereomeric mixture of the phenylglycinato complexes 3.1 $(\mathrm{R}=\mathrm{Ph})$ was separated by silica gel chromatography into three fractions: diastereopure $(C, S)-3.1$ (29 \% yield), a $80 / 20$ mixture enriched in the $(A, S)-3.1$ diastereomer $(34 \%)$ and a small amount (about $1.5 \%$ ) of diastereopure $(A, S)-3.1$ (see SI). ${ }^{9}$ The ${ }^{31} \mathrm{P}\left\{{ }^{1} \mathrm{H}\right\}$ NMR spectrum of the starting mixture and those of each diastereomer, once separated by chromatography, are shown in Figure 1. Diastereopure $(A, R)-\mathbf{3 . 1}$ can be alternatively prepared by using $(R)$-phenylglycine as chiral auxiliary according to Scheme 1.

The chiral auxiliary was replaced with two chlorido ligands, under retention of the configuration, ${ }^{10}$ by addition of excess of concentrated $\mathrm{HCl}_{\mathrm{aq}}$. From diastereopure $(C, S)$-3.1 the enantiopure dichlorido $(A)-\left[\mathrm{RhCl}_{2}\left(\kappa^{4} C, N, N^{\prime}, P-\mathbf{L}\right)\right]((A)-\mathbf{4})$ was obtained in more than $99.5 / 0.5$ enantiomeric ratio (e. r.). Figure 2 shows the HPLC traces of a racemic mixture of the dichlorido complexes $4,^{7 \mathrm{a}}$ as well as those of the enantiomers $(A)-4$ and $(C)-4 .{ }^{11}$ The circular dichroism spectra of both enantiomers are shown in Figure 2, too. No significant changes of the HPLC traces were observed when
Figure 2. HPLC traces of rac-, $A$ - and $C-4$ and $\mathrm{CD}$ spectra of $A$ (blue) and $\mathrm{C}-4$ (red) in $\mathrm{CH}_{2} \mathrm{Cl}_{2}$. enantiopure 4 was refluxed in methanol or heated at $130{ }^{\circ} \mathrm{C}$ in 2 ethoxyethanol, for 48 hours. Thus, it can be argued that the configuration at the metal is highly thermally stable.

We have recently reported that the chlorido ligands in $\mathrm{rac}-\mathbf{4}$ can be abstracted by silver cations, in some cases under severe conditions, affording racemic acetonitrilo or aquo complexes of formula $\left[\mathrm{Rh}\left(\kappa^{4} C, N, N^{\prime}, P-\mathbf{L}\right) \mathrm{A}(\mathrm{Solv})\right]\left[\mathrm{SbF}_{6}\right]_{\mathrm{n}}(\mathbf{1}, \mathbf{5 - 7}$, Table 1), in high yield. ${ }^{7 \mathrm{~b}}$ We have now found that this transformation occurs under retention of the configuration when enantiopure $(A)-\mathbf{4}$ was used. By this way, enantiopure samples of 1, 5-7 have been obtained.

Complexes 1, 5-7 catalyze the Diels-Alder (DA) reaction between methacrolein (MA) and HCp. In general, several hours are needed to get good conversions and enantiomeric ratios of up to $>$ $99 / 1$, in favor of the $R$ at C2 enantiomer, were achieved. Catalysts $\mathbf{1}$ and $\mathbf{5}$ were slightly more diastereo- and enantioselective than the chloride containing catalysts 6 and 7 .

Table 1. Selected data for the reaction between MA and HCp catalyzed by the complexes $1,5-7^{\mathrm{a}}$

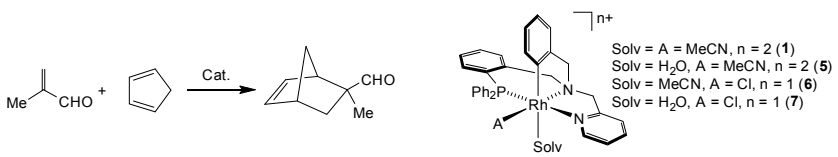

\begin{tabular}{|c|c|c|c|c|c|c|c|}
\hline Entry & Cat. & Cat.(\%) & $\mathbf{T}(\mathrm{K})$ & t (h) & $\begin{array}{l}\text { Conv. } \\
(\%)^{\mathbf{b}}\end{array}$ & exo/endo & $\begin{array}{l}\text { e. } r^{c, d} \\
(R / S)\end{array}$ \\
\hline 1 & \multirow{3}{*}{ (C)-1 } & \multirow{3}{*}{10} & 298 & 24 & 93 & $88 / 12$ & $91 / 9$ \\
\hline 2 & & & 273 & 70 & 47 & $87 / 13$ & $95 / 5$ \\
\hline 3 & & & 263 & 188 & 41 & $91 / 9$ & $95 / 5$ \\
\hline 4 & \multirow{3}{*}{$(A)-5$} & \multirow{3}{*}{10} & 298 & 22 & 90 & $89 / 11$ & $94 / 6$ \\
\hline 5 & & & 273 & 70 & 94 & $92 / 8$ & $97.5 / 2.5$ \\
\hline 6 & & & 263 & 164 & 93 & $93 / 7$ & $>99 / 1$ \\
\hline 7 & \multirow{3}{*}{$(A)-6$} & \multirow{3}{*}{10} & 298 & 24 & 79 & \multirow{2}{*}{$86 / 14$} & $80 / 20$ \\
\hline 8 & & & 273 & 70 & 24 & & $85 / 15$ \\
\hline 9 & & & 263 & 188 & 28 & $89 / 11$ & $85 / 15$ \\
\hline 10 & \multirow{4}{*}{ (A)-7 } & 5 & 298 & 96 & 92 & $85 / 15$ & $75 / 25$ \\
\hline 11 & & \multirow{3}{*}{10} & 298 & 23 & 90 & $85 / 15$ & $85 / 15$ \\
\hline 12 & & & 273 & 71 & 90 & $92 / 8$ & $91 / 9$ \\
\hline 13 & & & 263 & 187 & 55 & $89 / 11$ & $93 / 7$ \\
\hline
\end{tabular}

a Reaction conditions: catalyst, $1.16 \times 10^{-3} \mathrm{mmol}$; MA, 0.012 or $0.023 \mathrm{mmol}$; HCp, $0.081 \mathrm{mmol}$ and $10 \mathrm{mg}$ of $4 \AA \mathrm{MS}$, in $0.5 \mathrm{~mL}$ of $\mathrm{CD}_{2} \mathrm{Cl}_{2}$. ${ }^{\mathrm{b}}$ Based on MA. Determined by ${ }^{1} \mathrm{H} \mathrm{NMR} .{ }^{c}$ In the exo isomer. Determined by ${ }^{1} \mathrm{H}$ NMR with the chiral shift reagent $(+)$ $\mathrm{Eu}(\mathrm{hfc})_{3} .{ }^{\mathrm{d}}$ Absolute configuration of the major exo adduct $(R$ at the $\mathrm{C} 2$ ) established by comparison with literature data. ${ }^{12}$

The intermediates $\left[\mathrm{RhCl}\left(\kappa^{4} C, N, N^{\prime}, P-\mathbf{L}\right)(\mathrm{MA})\right]^{+}$and $\left[\mathrm{Rh}\left(\kappa^{4} C, N, N^{\prime}, P-\mathbf{L}\right)(\mathrm{NCMe})(\mathrm{MA})\right]^{+}$have been spectroscopically characterized (SI).

In both cases, considering the $A$ enantiomers, NOESY data are compatible only with the conformation in which the Si-face of the MA is shielded by the diphenylphosphino arm of the tetradentate ligand. Assumng this conformation as the reactive one, the reaction with $\mathrm{HCp}$ through the clear Re-face of the enal precisely suites the sign of the configuration of the major DA adduct obtained when enantiopure $(C)-\mathbf{1}$ and $(A)-\mathbf{5 - 7}$ were used as catalyst precursors.

On the other hand, ${ }^{1} \mathrm{H}$ and ${ }^{31} \mathrm{P}$ NMR measurements have been carried out in order to establish the rate determining step of the catalytic cycle. Under catalytic conditions MA is the limiting reagent and, once it has completely reacted, the resulting solution contains a mixture of exo and endo $\left[\mathrm{Rh}\left(\kappa^{4} C, N, N^{\prime}, P-\mathbf{L}\right) \mathrm{A}(\mathrm{DA}-\right.$ adduct $)]^{+\mathrm{n}}$ as the only metallic species. Upon addition of MA (2 
equiv.) to this solution, a fast exchange between the coordinated DA-adducts and free MA was observed, even below $263 \mathrm{~K}$ (the equilibrium was reached within aprox. $3 \mathrm{~min}$ ) while the concentration of residual $\mathrm{HCp}$ did not vary appreciably. Taking into account that, at $263 \mathrm{~K}$, the rate of the DA reaction is low (Table 1 ), the formation of the $\mathrm{C}-\mathrm{C}$ bonds should be the rate determining step.

The Gibbs free energy profile of the Diels-Alder reaction catalyzed by 6 (or 7) is shown in Figure 3. A similar profile was calculated when $\mathbf{1}$ or $\mathbf{5}$ are the catalysts (Figure S.7, SI). The MA complex $(A)-\left[\operatorname{RhCl}\left(\kappa^{4} C, N, N^{\prime}, P-\mathbf{L}\right)(\mathrm{MA})\right]^{+}$(XIa) forms upon reaction of $(A)-6$ with MA and thereafter reacts with HCp yielding the non-covalent adduct XIIa.

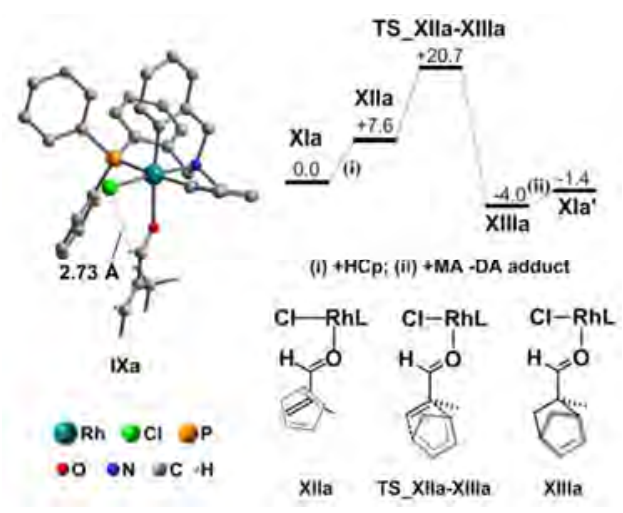

Figure 3. Gibbs free energy profile of the Diels-Alder reaction through the $R e$ enantioface of coordinated methacrolein in IXa. For brevity, only the profile for the reaction leading to the exo adduct is shown. Free energies are in $\mathrm{kcal} \cdot \mathrm{mol}^{-1}$.

The complex $(A)-\left[\operatorname{RhCl}\left(\kappa^{4} C, N, N^{\prime}, P-\mathbf{L}\right)(\mathrm{DA} \text {-adduct })\right]^{+}$(XIIIa) is obtained through the transition state TS_XIIa-XIIIa and finally XIa re-forms upon reaction of XIIIa with MA. The structure of XIa nicely fits in with the above mentioned spectroscopic data for $\left[\mathrm{RhCl}\left(\kappa^{4} C, N, N^{\prime}, P-\mathbf{L}\right)(\mathrm{MA})\right]^{+}$(vide supra). As a result of the $\mathrm{CH} \cdots \mathrm{Cl}$ short contact $(2.73 \AA)$ and of the s-trans arrangement of the $\mathrm{C}\left(\mathrm{sp}^{2}\right)-\mathrm{C}\left(\mathrm{sp}^{2}\right)$ single bond, the Re-face of MA is clear, while the other is shielded by one of the $\mathrm{PPh}$ groups (Figure 3). Accordingly, the attack of HCp to the Re-face exhibits a significantly lower barrier (exo, 20.7; endo $\left.22.7 \mathrm{kcal} \cdot \mathrm{mol}^{-1}\right)$ than that to the $\mathrm{Si}$ face (exo 24.9 ; endo $25.3 \mathrm{kcal} \cdot \mathrm{mol}^{-1}$, see SI) nicely fitting in with the formation of the $R$-DA adduct as the major product. Further, the exo attack is favored over the endo one, mostly kinetically and to less extent thermodynamically (SI). Regarding the enantioselectivity, it should also be mentioned that the minor $S$-DA adduct may also form -although to a smaller extent- as the result of the attack of the HCp to the clear Si-face of coordinated MA in less stable rotamers of $\left[\mathrm{RhCl}\left(\kappa^{4} C, N, N^{\prime}, P-\mathbf{L}\right)(\mathrm{MA})\right]^{+}$(see $\left.\mathrm{SI}\right)$.

In summary a satisfactory optical resolution of the rhodium complexes $\mathrm{rac}-\mathbf{1}$ into $(A) \mathbf{- 4}$ and (C)-4 has been achieved and, taking advantage of the coordinative stability and the configurational inertness of the tetradentate tripodal ligand $\mathbf{L}$, a family of enantiopure chiral-at-metal complexes of formula $\left[\mathrm{Rh}\left(\kappa^{4} C, N, N^{\prime}, P-\mathbf{L}\right) \mathrm{A}(\mathrm{Solv})\right]^{+\mathrm{n}}(\mathbf{1}, \mathbf{5 - 7})$ has been prepared. Notably, in all of them the DFT molecular models and, where appropriate, the crystal structures ${ }^{7 b}$ show that the ligand $\mathbf{L}$ molds a well-defined chiral pocket at the rhodium atom. Furthermore, when enantiopure 1, 5-7 were used as catalyst precursors in the DA reaction between MA and $\mathrm{HCp}$, the observed enantiomeric ratios in the DA adduct (up to $>99 / 1$ ) definitely point out that a high asymmetric induction can be achieved under catalytic conditions. Indeed, experimental NMR data and DFT calculations nicely show that one enantioface of the coordinated MA is shield- ed by the diphenylphosphino group while the other is clear. Thus, the preferential attack of $\mathrm{HCp}$ to the clear enantioface takes place rendering good-to-excellent enantiomeric ratios in the DA product.

The encountered metal configurational stability, the availability of two potentially labile sites at the metal center, the observed level of chiral induction and its origin make appealing and more than promising the use of the complexes reported herein in a number of catalytic asymmetric processes. On the other hand, the modular nature of $\mathbf{L}$, as well as the possible incorporation of other $d^{6}$ metal complexes opens the door to the exploitation of this type of chiral-at-metal catalysts in a broad variety of asymmetric transformations.

\section{ASSOCIATED CONTENT}

\section{Supporting Information}

The Supporting Information is available free of charge via the Internet at http://pubs.acs.org.

Further details of experimental metods, compound characterization, spectra, DFT calculations (PDF), crystallographic data (CIF).

\section{AUTHOR INFORMATION}

\section{Corresponding Authors}

dcarmona@unizar.es (D. C.); riromar@unizar.es, (R. R.); passarel@unizar.es (V.P.).

\section{ORCID}

María Carmona: 0000-0002-2343-0156

Ricardo Rodríguez: 0000-0002-8845-0174

Vincenzo Passarelli: 0000-0002-1735-6439

Fernando J. Lahoz: 0000-0001-8054-2237

Pilar García-Orduña: 0000-0002-7063-1292

Daniel Carmona: 0000-0003-4196-5856

\section{Notes}

The authors declare no competing financial interests.

\section{ACKNOWLEDGMENTS}

We thank the Ministerio de Economía y Competitividad of Spain (CTQ2015-66079-P) and Gobierno de Aragón (Grupo Consolidado E-63: Catalizadores Organometálicos Enantioselectivos) for financial support. M. C. acknowledges Diputación General de Aragón, CSIC and European Social Fund for a grant. R. R. acknowledges the Ministerio de Economía y Competitividad of Spain for a Ramón y Cajal (RYC-2013-13800) grant. P. G.-O. acknowledges CSIC, European Social Fund and Ministerio de Economía y Competitividad of Spain for a PTA contract. V. P. thanks the resources of the supercomputer "Memento" and the technical expertise and assistance provided by the Institute for Biocomputation and Physics of Complex Systems (BIFI), Universidad de Zaragoza.

\section{REFERENCES}

(1) (a) Comprehensive Asymmetric Catalysis; Jacobsen, E. N.; Pfaltz, A.; Yamamoto, H., Eds.; Vol I-III, Springer: New York, 1999; Suppl. 1 and 2, Springer: New York, 2004. (b) Catalytic Asymmetric Synthesis, Ojima, I., Ed.; Wiley-VCH: Weinheim, 2000. (c) Noyori, R. In Asymmetric Catalysis in Organic Synthesis, Wiley: Hoboken, 1994. (d) Walsh, P. J.; Kozlowski, M. C. In Fundamentals of Asymmetric Catalysis, University Science Books: Sausalito, 2009. (e) Lewis Acids in Organic Synthesis, Yamamoto H. Ed., Wiley-VCH: Weinheim, 2000. (f) Transition Metals for Organic Synthesis: Building Blocks and Fine Chemicals, $2^{\text {nd }}$ Edition, Beller, M.; Bolm, C., Eds.; Wiley-VCH: Weinheim, 2008. 
(2) (a) Zhang, L.; Meggers, E. Acc. Chem. Res. 2017, 50, 320-330. (b) Fontecave, M.; Hamelin, O.; Ménage, S. Top. Organomet. Chem. 2005, 15, 271-288.

(3) Zhang, L.; Meggers, E. Chem. Asian J. 2017, 12, 2335-2342 and references therein.

(4) (a) Bauer, E. B. Chem. Soc. Rev. 2012, 41, 3153-3167 and references therein. (b) Mukherjee, T.; Ganzmann, C.; Bhuvanesh, N.; Gladysz, J. A. Organometallics 2014, 33, 6723-6737. (c) Carmona, D.; Lamata, M. P.; Sánchez, A.; Viguri, F.; Rodríguez, R.; Oro, L. A.; Liu, C.; DíezGonzález, S.; Maseras, F. Dalton Trans. 2014, 43, 11260-11628. (d) Méndez, I.; Rodríguez, R.; Polo, V.; Passarelli, V.; Lahoz, F. J.; GarcíaOrduña, P.; Carmona, D. Chem. Eur. J. 2016, 22, 11064-11083.

(5) See for example: (a) Mezzetti, A. Dalton Trans. 2010, 39, 78517869. (b) Huber, R.; Bigler, R.; Mezzetti, A. Organometallics 2015, 34, 3374-3384. (c) Oguma, T.; Katsuki, T. in Transition Metal Catalysis in Aerobic Alcohol Oxidation, Ch. 9: Asymmetric Oxidation of Alcohols and Phenol Derivatives with Air as Oxidant; Cardona, F.; Parmeggiani, C.; From series: RSC Green Chemistry, RSC: London, 2015, pp. 231-255. (d) Li, Y.-Y.; Yu, S.-L.; Shen, W.-Y.; Gao, J.-X. Acc. Chem. Res. 2015, 48, 2587-2598. (e) Morris, R. H. Acc. Chem. Res. 2015, 48, 1494-1502. (f) Larrow, J. F.; Jacobsen, E. N. Top. Organomet. Chem. 2004, 6, 123-152. (g) Cussó, O.; Ribas, X.; Costas, M. Chem. Commun. 2015, 51, 14285 14298. (h) Hechavarría Fonseca, M.; König, B. Adv. Synth. Catal. 2003, 345, 1173-1185. (i) Sohtome, Y.; Nakamura, G.; Muranaka, A.; Hashizume, D.; Lectard, S.; Tsuchimoto, T.; Uchiyama, M.; Sodeoka, M. Nat. Commun. 2017, 8, 14875. (j) Ehnbom, A.; Ghosh, S. K.; Lewis, K. G.; Gladysz, J. A. Chem. Soc. Rev. 2016, 45, 6799-6811. (k) Ghosh, S. K.; Ehnbom, A.; Lewis, K. G.; Gladysz, J. A. Coord. Chem Rev. 2017, 350, 30-48. (1) Gong. L.; Chen, L.-A.; Meggers, E. Angew. Chem. Int. Ed. 2014, 53, 10868-10874. (m) Cao, Z.-Y.; Brittain, W. D. G.; Fossey, J. S.; Zhou, F. Catal. Sci. Technol. 2015, 5, 3441-3451. (n) Meggers, E. Angew. Chem. Int. Ed. 2017, 56, 5668-5675 and references therein.

(6) (a) Knof, U.; von Zelewsky, A. Angew. Chem. Int. Ed. 1999, 38, 302-322. (b) Knight, P. D.; Scott, P. Coord. Chem. Rev. 2003, 242, 125143. (c) Meggers, E. Eur. J. Inorg. Chem. 2011, 2911-2926.

(7) (a) Carmona, M.; Rodríguez, R.; Méndez, I.; Passarelli, V.; Lahoz, F. J.; García-Orduña, P.; Carmona, D. Dalton Trans. 2017, 46, 7332-7350. (b) Carmona, M; Tejedor, L.; Rodríguez, R.; Passarelli, V.; Lahoz. F. J.; García-Orduña, P.; Carmona, D. Chem. Eur. J. 2017, 23, 14532-14546.

(8) To simplify the notation of the stereochemical descriptors, we will label diastereomers $\left(\left(S_{\mathrm{N}}, S_{\mathrm{C}}\right)-O C-6-34-C\right)-3$ and $\left(\left(R_{\mathrm{N}}, S_{\mathrm{C}}\right)-O C-6-34-A\right)-3$ as $(C, S)-\mathbf{3}$ and $(A, S)-\mathbf{3}$, respectively. $S$ refers to the configuration at the stereogenic carbon of the amino carboxylato and $C$ and $A$ stand for the clockwise or anticlockwise arrangement of the donor atoms at the equatorial plane of the octahedral complex. This simplification will be properly applied to all the remaining chiral metallic complexes.

(9) In our hands, the chromatographic separation of other aminocarboxylato derivatives 3 was less efficient. Other tested chiral auxiliaries as mandelates, hexahydromandelates or tartrates did not provide the expected rhodium diastereomers or we were not able to separate them either by crystallization or by chromatography.

(10) Note that, due to the nomenclature rules, in this transformation, the retention of the configuration implies the change from $A$ to $C$, and vice versa, for the stereogenic metal descriptor.

(11) Enantiopure $(C)-\mathbf{4}$ was prepared from diastereopure $(A, S)$ - or $(A, R)-3.1$

(12) Furuta, K.; Shimizu, S.; Miwa, Y.; Yamamoto, H. J. Org. Chem. 1989, 54, 1481-1483. 
The Metal as Source of Chirality in Octahedral Complexes with Tripodal Tetradentate Ligands

María Carmona, Ricardo Rodríguez, Vincenzo Passarelli, Fernando J. Lahoz, Pilar García-Orduña and Daniel Carmona

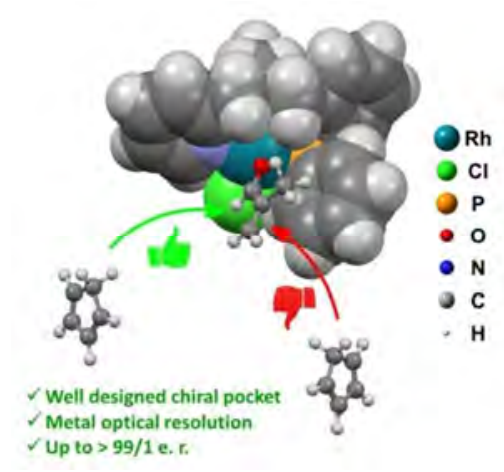

